\title{
Cadherins Glycans in Cancer: Sweet Players in a Bitter Process
}

\section{Sandra Carvalho, Celso A. Reis, and Salomé S. Pinho}

1-i3S - Instituto de Investigação e Inovação em Saúde, Universidade do Porto, Rua Alfredo Allen, 4200-135 Porto, Portugal; Institute of Molecular Pathology and Immunology of the University of Porto (IPATIMUP), Rua Júlio Amaral de Carvalho 45, 4200-465 Porto, Portugal.

2-i3S - Instituto de Investigação e Inovação em Saúde, Universidade do Porto, Rua Alfredo Allen, 4200-135 Porto, Portugal; Institute of Molecular Pathology and Immunology of the University of Porto (IPATIMUP), Rua Júlio Amaral de Carvalho 45, 4200-465 Porto, Portugal; Institute of Biomedical Sciences of Abel Salazar (ICBAS), University of Porto, Rua de Jorge Viterbo Ferreira, 4050-313 Porto, Portugal; Medical Faculty, University of Porto, Alameda Professor Hernâni Monteiro, 4200-319 Porto, Portugal.

3-i3S - Instituto de Investigação e Inovação em Saúde, Universidade do Porto, Rua Alfredo Allen, 4200-135 Porto, Portugal; Institute of Molecular Pathology and Immunology of the University of Porto (IPATIMUP), Rua Júlio Amaral de Carvalho 45, 4200-465 Porto, Portugal; Medical Faculty, University of Porto, Alameda Professor Hernâni Monteiro, 4200-319 Porto, Portugal. Electronic address: salomep@ipatimup.pt.

Originally published in Trends Cancer. 2016 Sep;2(9):519-531. doi: 10.1016/j.trecan.2016.08.003. 


\section{ABSTRACT}

Cadherins are key components in tissue morphogenesis and architecture, contributing to the establishment of cohesive cell adhesion. Reduced cellular adhesiveness as a result of cadherin dysfunction is a defining feature of cancer. During tumor development and progression, major changes in the glycan repertoire of cancer cells take place, affecting the stability, trafficking, and cell-adhesion properties of cadherins. Importantly, the different glycoforms of cadherins are promising biomarkers, with potential clinical application to improve the management of patients, and constitute targets for the development of new therapies. This review discusses the most recent insights on the impact of glycan structure on the regulation of cadherin function in cancer, and provides a perspective on how cadherin glycans constitute tumor biomarkers and potential therapeutic targets.

\section{Cadherin Glycans: More Than Sweet Molecules in Cancer}

Loss of cell adhesion is a major molecular event occurring during malignant transformation [1]. Concomitantly, cancer cells suffer profound alterations in the biological repertoire of glycans attached to the cell surface and/or expressed in the cytoplasm [2]. Glycans exert a powerful effect on cadherins by influencing their biophysical properties, homotypic interactions, and cell adhesion functions. The type of glycan structure has a differential impact on cadherin-mediated cell adhesion, thereby regulating cancer cell fate. For example, cadherins bearing branched complex Nglycans are less competent in terms of cell adhesion and are associated with cancer progression. By contrast, the expression of bisecting glycans on cadherins strengthens cell adhesion and is associated with cancer suppression. In this review we present the most recent evidence of the roles that different glycans play in the regulation of cadherin function in cancer, and discuss applications in oncology.

\section{Cadherins}

The cadherin superfamily (see Glossary) comprises a set of calcium-dependent adhesion molecules that participate in cell-cell interactions and determine cell polarity and tissue archi- tecture during embryonic morphogenesis and homeostasis [3,4]. These adhesion glycoproteins organize into lateral clusters at the cell surface where they mediate homophilic contacts between neighboring cells and participate in dynamic interactions with the actin cytoskeleton as core components of the adherens junctions (Box 1) [5]. In fact, classical cadherins are membrane- spanning macromolecular complexes that serve as important mechanosensors at cell-cell junctions, modulating cell behavior during tissue remodeling and cellular transformation $[6,7]$.

Dysfunction of cadherins is an important determinant of cancer development and progression $[1,8]$. The disruption of cadherin-mediated cell adhesion leads to disorganized tissue architecture $[9,10]$. Because many solid tumors are of epithelial origin, epithelial cadherin (E-cadherin or cadherin 1 ) is an attractive target in cancer. E-cadherin is a tumor suppressor [11] that can be dysregulated by multiple mechanisms including loss of heterozygosity [12,13], inherited and somatic mutations $[14,15]$ (well characterized in gastric cancer), promoter hyper- methylation $[16,17]$, transcriptional silencing [18], endocytosis [19,20], and proteolysis [21]. However, a high percentage of human epithelial invasive cancers with dysfunction of E-cadherin- mediated cell adhesion do not have 
genetic and structural alterations in the protein, and the underlying mechanism of cadherin dysregulation remains unknown [22]. This gap in knowledge has fuelled the identification of other molecular mechanisms of (dys)regulation of cadherins in cancer. Glycosylation has been pinpointed as a fundamental mechanism controlling the adhesion functions of cadherins, and has potential to be targeted in cancer therapy $[23,24]$.

Protein glycosylation is an important post-translational modification in eukaryotes, where more than half of all proteins are glycosylated [25]. The structural diversity of glycans that can be added to proteins - known as the glycome or the glycan repertoire - contributes to a wide range of biological functions $[26,27]$. In cancer, cellular transformation is typically accompanied by changes in the glycome, and aberrant glycosylation is considered to be a hallmark of neoplastic cells $[2,28-$ 30].

Decoding the function of cadherin glycans in cancer has been revealed to be fundamental for further understanding cancer cell biology and to potentially improve the clinical management and treatment of patients.

Box 1. E-Cadherin Structure

The immature form of E-cadherin is a polypeptide composed of a propeptide sequence of about 130 amino acids (aa) and a mature polypeptide of about 728 aa. The propeptide corresponds to a short signal sequence for import into the ER where it undergoes cytoplasmic trimming [11]. Following this trimming process, mature E-cadherin is routed towards the basolateral surface of epithelial cells. Ecadherin mature protein is organized into three major structural domains: an $\mathrm{N}$ - terminal ectodomain of about 550 aa, a single transmembrane domain, and a short C-terminal cytoplasmic domain of about 150 aa [11]. The ectodomain comprises five extracellular cadherin (EC) repeat domains that mediate adhesive (trans) interactions with cadherins embedded in opposing membranes, which then undergo lateral (cis) interactions with adjacent cadherin molecules $[136,137]$. The cadherin cytoplasmic domain interacts with the so-called 'core cadherin- catenin complex' (b-catenin, p120-catenin, and many others) which in turn provides anchorage to the actin cytoskeleton to form stable cell-cell contacts. The dynamic cooperation of cadherin-catenin complexes with the cytoskeleton contributes to both the stability and plasticity of adherens junctions [138-140].

\section{Glycosylation: Key Mechanism in Cadherin-Mediated Cell Adhesion.}

The regulatory power of glycans is evident in the early secretory pathway of cadherins. Glycosylation of cadherins starts in the endoplasmic reticulum (ER) while the proteins emerge from the translocon complex (Box 2) [31]. The addition of a core-oligosaccharide structure to asparagine (Asn) confers thermodynamic stability [32], guides correct folding [33], and ensures structural assembly and stability of the proteins [34]. The trafficking of cadherins to the Golgi is also controlled by glycosylation through the cytoplasmic modification of cadherin with O-linked $\mathrm{N}$ acetylglucosamine (O-GlcNAc) structures (Figure 1) [35]. The O-GlcNAcylation modification of Ecadherin is associated with its retention in the ER by interfering with E-cadherin binding to p120- 
catenin and type I g-phosphatidylinositol phosphate kinase (PIPKIg), a protein required for recruitment of E-cadherin to adhesion sites [36].

Glycans also dictate spatial cadherin arrangement at the cell membrane and contribute to homotypic cellular interactions. E-cadherin modified with high-mannose $\mathrm{N}$-glycans is predominantly expressed at the cell surface and more effectively enhances cell-cell interactions than does E-cadherin modified with hybrid and complex N-glycans [37-39]. Interestingly, glycans modulate lateral (cis) interactions between neural cadherins ( $\mathrm{N}$-cadherins or cadherin 2) on the same cell, thereby governing the dynamics of intercellular junction assembly [40-42]. It is important to mention that this dynamic contribution of glycans to the regulation of cadherin- mediated cell adhesion is known to be cell- and tissue-specific.

Recent reports have identified cadherins as major carriers of O-linked mannose structures, and these O-mannose glycans on cadherins are not further elongated (Figure 1) [43,44]. Omannosylation-deficient embryos exhibit defects in the molecular architecture of cell-cell contact sites and are arrested at the morula-to-blastocyst transition stage [45]. Conserved $\mathrm{O}$ mannosylation sites in $\mathrm{T}$ - (cadherin $13, \mathrm{H}$-cadherin), $\mathrm{E}$-, and $\mathrm{N}$-cadherins across different species suggest a general role of O-mannosyl glycans in the regulation of cadherin-mediated cell adhesion $[43,46]$. In fact, inhibition of O-mannosylation leads to compromised cadherin- mediated adhesion [45].

Regarding N-glycosylation, there are four potential N-glycosylation sites (Asn-554, Asn-566, Asn618, and Asn-633) in the ectodomain of E-cadherin (Figure 1), and the occupancy of each site by a specific N-glycan depends on the cell, tissue, and pathophysiological context [47]. Proliferating epithelial cells (sparse cell cultures) display E-cadherin modified with complex type N-glycans, leading to unstable adherens junctions, while differentiated cells with mature adhe- rens belts (dense cell cultures) have E-cadherin modified with hybrid/high-mannose oligosaccharides [48]. Nglycosylation also influences the molecular organization of adherens junctions and anchorage to the actin cytoskeleton [48,49], indirectly impacting on the assembly of tight junctions [50] 
Box 2. Protein Glycosylation in Homeostasis and Cancer

Glycomics defines the structure and functional roles of glycans in complex biological systems and can be applied to understanding how post-translational modifications via glycosylation affect cancer development and progression. The mammalian glycome repertoire is estimated to be 10104 times larger than the proteome, and is far more complex than either the genome or the proteome [141]. Glycosylation consists of the covalent attachment of carbohydrate structures to proteins and lipids in the endoplasmic reticulum (ER) and Golgi, producing different families of glycoconjugates [2]. In proteins, glycans are usually attached to the polypeptide backbone via N-or O- linkages.

The $\mathrm{N}$-linked glycans are attached to asparagine (Asn) residues of nascent proteins within the consensus peptide sequence Asn-X-Ser/Thr, where $\mathrm{X}$ is any amino acid except proline [82]. N-linked glycans are further extended and elongated in the Golgi compartment by several glycosyltransferases that operate in a stepwise manner. $\mathrm{N}$-glycosylation initiates at the ER membrane where Glc3MangGlcNAc2 is synthesized and covalently coupled to the polypeptide backbone. This en bloc transfer is catalyzed by oligosaccharyltransferase (OST), a multisubunit protein complex associated with the translocon complex. At the ER, glycans have a common role in protein folding, quality control, and some sorting events [33]. Once glycoproteins are folded and oligomerized correctly, they move to the Golgi, where N-glycans are further extensively modified $[82,142]$. During elongation and extension at the trans-Golgi, several carbohydrate modifications can occur: (i) core fucosylation, mediated by Fut8; (ii) elongation of branch $\mathrm{N}$-acetylgluco- samine (GlcNAc) residues of $\mathrm{N}$-glycans (e.g., poly-N-acetyllactosamine, polyLacNAc); or (iii) or 'capping' and 'decoration' of elongated branches (the addition of sialic acid, Neu5Ac; fucose, Fuc; galactose, Gal; or N-acetylgalactosamine, GalNAc) [82].

Cancer cells exhibit a predominance in b1,6-GlcNAc residues (catalyzed by $\mathrm{N}$ acetylglucosaminyltransferase $\mathrm{V}, \mathrm{GnT}-\mathrm{V}$ ) that are frequently extended with polylactosamine structures (Galb1,4GlcNAcb1,3, a ligand for galectins), which in turn are terminally modified with sialic acid (Figure I). These glycan structures impair the function of cadherins and promote malignant and invasive phenotypes. Normal/benign cells tend to display E-cadherin modified with bisecting GlcNAc residues (catalyzed by N-acetylglucosaminyltransferase III, GnT-III), with negligible levels of branched glycans.

O-linked glycosylation consists in the attachment of carbohydrate moieties to serine (Ser) or threonine (Thr) residues [82]. Mucin-type O-glycosylation consists of the attachment of GalNAc structures to Ser or Thr residues [143]. O-mannosyla- tion is initiated by the covalent attachment of mannose to Ser or Thr residues of secretory and membrane proteins in the ER lumen, catalyzed by the protein O-mannosyltransferases 1 and 2 (POMT1 and POMT2) [144]. The O-mannose core structures can be further extended via GlcNAcb1-2Man, GlcNAcb1-4Man, or GlcNAcb1-6Man linkages [145]. In homeostasis, cadherins are O-mannosylated. During malignant transformation cadherins show decreased O-manno- sylation concomitant with increased branched structures [87].

Several factors may affect glycan heterogeneity in proteins: the expression and localization of glycosyltransferases in the ER/Golgi complex, the ratio of enzymatic activities, enzyme accessibility to substrate, nucleotide sugar metabolism, and Golgi $\mathrm{pH}[2,29]$, and the variability of glycosylation patterns precludes the precise prediction of glycans in a particular cell type. 
Owing to the complexity and dynamic nature of glycans, their biological roles are varied and range from the folding of nascent proteins and intracellular trafficking to roles in molecular and cellular homeostasis [146], cell adhesion and cell signaling [147], immune modulation, and endocytosis $[148,149]$.

\section{Glycosylated Cadherins in Cancer Development}

The glycosylation pattern of cadherins undergoes profound modifications during the acquisition of the malignant phenotype (Figure 2, Key Figure) $[28,51]$. The most-common glycosylation changes are altered core fucosylation, highly branched $\mathrm{N}$-glycans, increased terminal sia- lylation, and altered expression of O-mannose structures $[23,29]$.

Core fucosylation consists of the addition of 11,6 -fucose to the innermost $\mathrm{N}$-acetylglucosamine (GlcNAc) residue of the oligosaccharide core, catalyzed by /-1,6-fucosyltransferase (Fut8) (Box 2). In gastric cancer, core fucosylation is downregulated, and its upregulation was found to inhibit cell proliferation of human gastric cancer cells $[52,53]$. In colon cancer cells, introduction of Fut 8 results in increased expression of E-cadherin at the cell surface [54]. Evidence suggests that addition of 11,6 -fucose to E-cadherin reduces tyrosine (Tyr)-654 phosphorylation of b-catenin, leading to reduced accumulation of nuclear b-catenin and thus to increased binding affinity to E-cadherin and enhancement of cell-cell adhesion [55]. Recently, it was reported that decreased core fucosylation of E-cadherin in lung cancer cells activates Src kinase, inducing an upregulation of $\mathrm{N}$-cadherin and Snaily, two molecular drivers of the epithelial-mesenchymal transition (EMT) [56]. However, the role of core fucosylation in the regulation of E-cadherin function remains controversial because increased core fucosylation of E-cadherin is also observed during lung cancer progression [57].

b1,6-N-Acetylglucosaminyltransferase-V (GnT-V) is involved in the biosynthesis of the so-called 'b1,6-GlcNAc branch' structure of N-glycans (Box 2) [58], and is highly implicated in tumor progression [51]. In fact, transcription of MGAT5 (gene encoding GnT-V) is upregulated by oncogene signaling $[58,59]$. Several types of cancer exhibit overexpression of b1,6-GlcNAc branched N-glycans, such as gastric [6o], colon [61], and breast tumors [62]. GnT-V expression impairs epithelial cell contact inhibition [63], increases cell proliferation in normal mammary epithelial cells [64], and modulates the canonical Wnt/b-catenin and Her-2-mediated signaling pathways [65]. Suppression of GnT-V activity results in reduced levels of tumor-initiating cells and tumorigenic potential in vivo and in vitro $[66,67]$, as wells as delayed tumor onset [64]. Further extension of $\mathrm{b}_{1}, 6-\mathrm{GlcNAc}$ branched $\mathrm{N}$-glycans with poly-N-acetyllactosamine structures potentiates their binding to galectins (a family of carbohydrate-binding proteins) that generate the lattices (galectin-glycan structures) controlling the surface retention time of gly- coproteins $[68,69]$. Modification of E-cadherin with b1,6-GlcNAc branched N-glycans affects its cell-membrane expression [70] and the molecular assembly and stability of adherens junctions [71] by interfering with the recruitment of b-catenin and p120-catenin, thereby compromising their clustering capacity at the cell surface [72] and reducing the cellular adhesion [73] (Figure 2). Branched N-glycosylation on $\mathrm{N}$-cadherin affects the outside-in signal transduction pathway of ERK and reduces homotypic cell-cell adhesion $[42,74]$. 
Glycosylation of cadherins during cancer development occurs in a site- and context-specific manner. Each site can be differently glycosylated, not all glycosylation sites are occupied, and not all sites are important for cadherin-mediated adhesion in all contexts. For instance, in human breast carcinomas, all four potential $\mathrm{N}$-glycosylation sites of $\mathrm{E}$-cadherin are glycosylated and each $\mathrm{N}$ glycan has distinctive functions. N-glycans at Asn-554 and Asn-566 affect cell-cycle progres- sion and proliferation through the activation of the ERK signaling pathways [75], as well as adhesion and the composition of adherens junctions [76]. Removal of specific N-glycans from E- cadherin increases tyrosine phosphorylation of b-catenin, destabilizing the adhesion complex [76]. In oral cancer, hyperglycosylation at Asn-554 with complex N-glycans, as a result of overexpression of DPAGT 1 and activation of Wnt signaling, reduces cell adhesion and com- promises the intercellular junctions [77,78]. Glycan at Asn-633 is important for E-cadherin expression, folding, and trafficking to the Golgi because deglycosylation at this site specifically arrests E-cadherin in the calnexin/calreticulin quality-control cycle at the ER, where it is recognized as a misfolded protein and then degraded via the ER-associated protein degradation (ERAD) pathway [79]. In gastric cancer cells, only Asn-554 and Asn-633 were demonstrated to be modified with b1,6-GlcNAc branched $\mathrm{N}$-glycans and high mannose/hybrid N-glycans, respec- tively. Interestingly, preventing glycan modification at Asn-554, by blocking GnT-V-mediated N- glycosylation, resulted in increased expression of E-cadherin at the cell surface and increased cis- dimerization capacity, thereby having a protective effect on E-cadherin adhesion function [72].

Overexpression of sialylated structures is another cancer glycophenotype [80]. The addition of sialic acid residues, which frequently terminate the carbohydrate chains, is mediated by sialyltransferases, a family of glycosyltransferases frequently upregulated in cancer [81]. Sialic acids can be attached to subterminal sugars such as $\mathrm{N}$-acetylgalactosamine or $\mathrm{N}$-acetylglucos- amine (through an / $2-6$ bond), to galactose (through /2-3 or / $2-6$ bonds), or to another terminal sialic acid (through an $/ 2-8$ bond), thereby generating polysialic acids $[82,83]$. The position of sialylated antigens on the cell membrane can regulate glycoprotein conformation, clustering, and cell-cell interactions such as adhesion. Electrostatic repulsion of negative charges on sialic acids disrupts cellular adhesion by breast carcinoma cells $[84,85]$ and promotes their tumor-initiating potential [86].

Cancer cells are also characterized by reduced protein O-mannosylation. Loss of O-mannosyl glycans attached to E-cadherin affects E-cadherin expression and compromises cadherin- catenin interactions. Interestingly, reduced GnT-V-mediated N-glycosylation of E-cadherin is accompanied by increased O-mannosylation [87].

\section{Glycosylated Cadherins in Invasion and Metastasis}

Functional changes in the glycan repertoire play significant roles in oncogenic transformation by potentiating invasion and the metastatic behavior of cancer cells [2]. Despite the huge diversity of glycans, only a specific set of cadherins glycoforms are associated with metastasis (Figure 2).

O-GlcNAcylation modification is linked to cellular features relevant to metastasis in ovarian and prostate cancer cells $[88,89]$. In fact, increased O-GlcNAcylation in cancer reduces the expression of E-cadherin, suggesting increased retention of E-cadherin in the ER. Core fucosylation and sialylation are significantly increased in the serum N-glycome of cancer patients [90], and the 
increase is associated with invasion [91,92]. The fucosylation index of E-cadherin is considered as a potential prognostic marker of metastatic lung adenocarcinoma [57]. Expression of sialyl Lewis $X$ (SLe $\mathrm{x}$ ) in cancer is associated with invasion and poor outcome, and is accompanied by reduced levels of E-cadherin [84,93-95] and an altered pattern of E-cadherin localization in pancreatic adenocarcinoma cells [96].

GnT-V is upregulated in invasive/metastatic cancer cells. GnT-V substrate proteins are a unique subset of coexpressed tumor markers associated with poor outcome in cancer patients [97]. In fact, downregulation of GnT-V in gastric cancer cells was described to inhibit metastasis via an EGFR signaling-initiated EMT phenotype and MMP-9 expression $[60,98]$. Expression of other metalloproteinases is also modulated by GnT-V, and is thus associated with increased invasive potential of colon cancer cells [99,100]. By contrast, upregulation of GnT-V increases the expression of the EMT-associated factors snail, twist, and N-cadherin, and induces changes in E-cadherin localization [101]. Specific modification of E-cadherin with b1,6-GlcNAc branched N-glycans in invasive gastric carcinomas correlates with poor survival rates of patients [72].

\section{Glycosylated Cadherins and Cancer Suppression}

N-Acetylglucosaminyltransferase-III (GnT-III), encoded by MGAT3, catalyzes the addition of a GlcNAc residue to the b-mannose via a b1,4 linkage $[58,102]$ (Box 2). The resulted 'bisecting GlcNAc' structure impacts on the activities of other glycosyltransferases by restricting the $\mathrm{N}$ - glycan conformation [103,104], thus preventing further processing and elongation of $\mathrm{N}$-glycans such as GnT-V-mediated branching $[105,106]$. GnT-III activity was reported to antagonize GnT-V effects on cell survival and metastasis. Whereas GnT-III (Mgat3)-deficient mice showed increased tumor growth and metastasis [107], upregulation of GnT-III in highly-metastatic mouse melanoma cells precludes GnT-V activity and results in significant suppression of cancer metastasis [108]. This suppressive role was attributed to GnT-III-mediated $\mathrm{N}$-glycosylation of E-cadherin. The overexpression of GnT-III leads to increased modification of E-cadherin with bisecting GIcNAc Nglycans, and this stabilizes the localization of E-cadherin at the cell surface [70] and delays its turnover rate and endocytosis [71]. As a result, the phosphorylation levels of b-catenin decrease and binding to E-cadherin at the cell surface is promoted [109], further contributing to stabilization of the adhesion complex and enhancement of intercellular binding [110].

On the other hand, E-cadherin-mediated adhesion complexes regulate the cellular levels of GnT- III. GnT-III expression is upregulated by increased cell-cell interactions via stable E-cadherin- cateninactin complexes $[70,111,112]$, and is downregulated when b-catenin translocates to the nucleus and activates Wnt signaling [113]. TGF-b-mediated EMT is also implicated in the regulation of GnT-III by promoting hypermethylation of MGAT3 and decreased expression and activity of GnT-III in epithelial cell lines $[114,115]$.

\section{Cadherin Glycans: Promising Tumor Biomarkers}

Tumor biomarkers are urgently needed to improve early diagnosis, prognosis, risk stratification, to predict therapeutic response, and to fuel the development of new drug targets and therapies [116]. Early dysregulation of E-cadherin-mediated cell-cell adhesion is a prominent alteration at the 
invasive fronts of many epithelial cancers, and is considered to be an early molecular event in diffuse-type gastric carcinoma [117] and lobular breast cancer [118]. Therefore, the underlying molecular alterations of cadherins dysfunction are promising biomarkers of tissue transformation and cancer progression. Given the profound alterations of the cadherin glycosignature and their correlation with the clinicopathological features of cancer patients, the clinical assessment of cadherin glycoforms either in tumor biopsies or in serum provides highly-specific glyco-based biomarkers for improving the clinical management of patients (Figure 2). As an example, it is currently possible to detect E-cadherin specifically modified with b1,6-GlcNAc branched N-glycans by in situ proximity ligation assay in gastric cancer biopsies $[71,72]$. Further correlation with the clinicopathological features revealed an association between this cadherin glycoform and poor prognosis of cancer patients [72].

Moreover, soluble cadherins resulting from the shedding of the cadherin ectodomain can also be detected in serum [119]. As a result of alterations in cellular topography during cancer cell transformation [120], soluble E-cadherin is constitutively shed at higher levels at tumor sites and metastatic foci [121]. Chan et al. showed that soluble E-cadherin levels were significantly elevated in patients with gastric cancer compared to healthy individuals [122]. Therefore, soluble E-cadherin specifically modified with b1,6-GlcNAc branched N-glycans can be used as a specific and noninvasive biomarker for the early detection of cancer and for monitoring tumor recurrence and therapeutic responses.

Glycomics and glycoproteomics-based technologies have been growing fast and are available to improve personalized medicine $[123,124]$. Rapid screening methods have been used to analyze glycosylation patterns on glycoproteins in large cohorts of patients, enabling the identification of a new generation of disease (glyco)biomarkers [125]. Profiling glycans in serum can be carried out by advanced (glyco)technological approaches such as mass spectrometry [126], lectin array [127-129], or via specific anti-glycan antibodies [130]. In addition, tumor-associated carbohy- drate antigens are known to elicit an immune response, raising the levels of serum antibodies that can be detected by printed glycan arrays [131]. Furthermore, glycan microarrays have important biomedical applications, including the characterization of potential inhibitors of glycosyltransfer- ases and glycosidases and new antagonists targeting specific lectin receptors $[132,133]$. Inte- grating these high-throughput glycotechnology methods in the clinical identification of cadherins glycoforms will certainly contribute to the discovery of a new era of cancer biomarkers paving the way for the development of new glycoprotein-based therapeutic drugs.

\section{Concluding Remarks and Future Directions}

Tumor development and progression are often associated with cadherin dysfunction and transition to a more motile and invasive phenotype. Glycosylation is a major post-translational modification of proteins that controls the biological function of cadherins in cancer. Under- standing how different glycan structures regulate and control the folding, trafficking, and cell- adhesion functions of cadherins will contribute greatly to elucidating the molecular pathogen- esis of cancer. With the advent of new and cutting-edge glycotechnological approaches able to detect different protein glycoforms with high specificity and sensibility [134], the specific detection of cadherin glycoforms in the serum of cancer patients constitutes a valuable non-invasive biomarker of prognostic value to be included in the clinical algorithm for cancer patients. Particularly, in diffuse-type gastric cancer

西 
and lobular breast cancer, where early alterations of E-cadherin triggers cancer development, the early in situ detection of cadherin alterations by assessing cadherin glycoforms is of great interest in improving early diagnosis and risk stratification of patients. Taken together, the detection of cadherin glycoforms is of major interest in oncology not only as a prognostic biomarker but also as potential target for the development of new therapies (see Outstanding Questions). Blocking the addition of the deleterious glycans, such as b1,6-GlcNAc branched N-glycans or sialylated glycans, on cadherin by using molecules targeting specific glycosyltransferases expressed in specific cells [135] constitutes an important strategy for modulating cancer cell behavior. Moreover, in the era of cancer immunotherapy, anticancer vaccines targeting tumor-associated carbohydrate antigens (such as cadherin glycoforms) provide another appealing option for boosting antitu-mor immunity. Activation of an antitumor immune response against cadherins bearing branched/sialylated glycans is foreseen as potential strategy for triggering tumor cell elimination.

\section{Acknowledgements}

IPATIMUP integrates the ${ }_{3} \mathrm{~S}$ Research Unit, which is partially supported by FCT, the Portuguese Foundation for Science and Technology (Fundação para a Ciência e a Tecnologia/Ministério da Ciência, Tecnologia e Inovação). This work was financed by FEDER - Fundo Europeu de Desenvolvimento Regional funds through the COMPETE 2020 - Operational Program for Competitiveness and Internationalization (POCl), Portugal 2020, and by Portuguese funds through the FCT in the framework of the project 'Institute for Research and Innovation in Health Sciences' (POCI-01-0145-FEDER-007274), PTDC/DTP-PIC/0560/2014, and PTDC/BBB-EBI/0567/2014. S.C. also acknowledges funding from the FCT (SFRH/BD/77386/2011). 


\section{REFERENCES}

1. Jiang, W.G. et al. (2015) Tissue invasion and metastasis: molec- ular, biological and clinical perspectives. Semin Cancer Biol. 35 (Suppl.), S244-S275

2. Pinho, S.S. and Reis, C.A. (2015) Glycosylation in cancer: mech- anisms and clinical implications. Nat. Rev. Cancer 15, 540-555

3. Vestweber, D. (2015) Cadherins in tissue architecture and dis- ease. J. Mol. Med. 93, 5-11

4. Niessen, C.M. et al. (2011) Tissue organization by cadherin adhesion molecules: dynamic molecular and cellular mecha- nisms of morphogenetic regulation. Physiol. Rev. 91, 691-731

5. Padmanabhan, A. et al. (2015) Jack of all trades: functional modularity in the adherens junction. Curr. Opin. Cell Biol. 36, 32-40

6. Hoffman, B.D. and Yap, A.S. (2015) Towards a dynamic under- standing of cadherin-based mechanobiology. Trends Cell Biol. 25, 803-814

7. Leckband, D.E. and de Rooij, J. (2014) Cadherin adhesion and mechanotransduction. Annu. Rev. Cell Dev. Biol. 30, 291-315

8. Gheldof, A. and Berx, G. (2013) Cadherins and epithelial-to- mesenchymal transition. Prog. Mol. Biol. Transl. Sci. 116, 317-336

9. van Roy, F. (2014) Beyond E-cadherin: roles of other cadherin superfamily members in cancer. Nat. Rev. Cancer 14, 121-134

10. Berx, G. and van Roy, F. (2009) Involvement of members of the cadherin superfamily in cancer. Cold Spring Harb. Perspect. Biol. 1, ao03129

11. van Roy, F. and Berx, G. (2008) The cell-cell adhesion molecule E-cadherin. Cell Mol. Life Sci. 65, $3756-3788$

12. Sang-Hyuk Lee, S.H. et al. (2011) Loss of heterozygosity of tumor suppressor genes (p16, Rb, Ecadherin, p53) in hypo- pharynx squamous cell carcinoma. Otolaryngol. Head Neck Surg. 145, 64-70

13. Palacios, J. et al. (2003) Frequent E-cadherin gene inactivation by loss of heterozygosity in pleomorphic lobular carcinoma of the breast. Mod. Pathol. 16, 674-678

14. van der Post, R.S. et al. (2015) Hereditary diffuse gastric cancer: updated clinical guidelines with an emphasis on germline $\mathrm{CDH}_{1}$ mutation carriers. J. Med. Genet. 52, 361-374

15. Corso, G. et al. (2014) E-Cadherin germline mutation carriers: clinical management and genetic implications. Cancer Metasta- sis Rev. 33, 1081-1094 
16. Shargh, S.A. et al. (2014) Downregulation of E-cadherin expres- sion in breast cancer by promoter hypermethylation and its relation with progression and prognosis of tumor. Med. Oncol. 31,250

17. Caldeira, J.R. et al. (2006) $\mathrm{CDH}_{1}$ promoter hypermethylation and $\mathrm{E}$-cadherin protein expression in infiltrating breast cancer. BMC Cancer 6, 48

18. Mazda, M. et al. (2011) E-Cadherin is transcriptionally activated via suppression of ZEB1 transcriptional repressor by small RNA- mediated gene silencing. PLoS ONE 6, e28688

19. Hirata, E. et al. (2014) Retrograde flow of cadherins in collective cell migration. Nat. Cell Biol. 16, $621-623$

20. Mellman, I. and Yarden, Y. (2013) Endocytosis and cancer. Cold Spring Harb. Perspect. Biol. 5, a016949

21. Hu, Q.P. et al. (2016) Beyond a tumor suppressor: soluble E- cadherin promotes the progression of cancer. Int. J. Cancer 138, 2804-2812

22. Corso, G. et al. (2013) Somatic mutations and deletions of the E-cadherin gene predict poor survival of patients with gastric cancer. J. Clin. Oncol. 31, 868-875

23. Pinho, S.S. et al. (2013) Gastric cancer: adding glycosylation to the equation. Trends Mol. Med. $19,664-676$

24. Zhao, Y. et al. (2008) Branched N-glycans regulate the biologi- cal functions of integrins and cadherins. FEBS J. 275, 1939-1948

25. Moremen, K.W. et al. (2012) Vertebrate protein glycosylation: diversity, synthesis and function. Nat. Rev. Mol. Cell Biol. 13, 448-462

26. Bard, F. and Chia, J. (2016) Cracking the glycome encoder: signaling, trafficking, and glycosylation. Trends Cell Biol. 26, 379-388

27. Hart, G.W. (2013) Thematic minireview series on glycobiology and extracellular matrices: glycan functions pervade biology at all levels. J. Biol. Chem. 288, 6903-6903

28. Munkley, J. and Elliott, D.J. (2016) Hallmarks of glycosylation in cancer. Oncotarget Published online March 17, 2016. http://dx. doi.org/10.18632/oncotarget.8155

29. Stowell, S.R. et al. (2015) Protein glycosylation in cancer. Annu. Rev. Pathol. 10, 473-510

30. Reis, C.A. et al. (2010) Alterations in glycosylation as biomarkers for cancer detection. J. Clin. Pathol. 63, 322-329

31. Cherepanova, N. et al. (2016) N-Linked glycosylation and homeostasis of the endoplasmic reticulum. Curr. Opin. Cell Biol. 41, 57-65 
32. Shental-Bechor, D. and Levy, Y. (2008) Effect of glycosylation on protein folding: a close look at thermodynamic stabilization. Proc. Natl. Acad. Sci. U.S.A. 105, 8256-8261

33. Xu, C. and Ng, D.T.W. (2015) Glycosylation-directed quality control of protein folding. Nat. Rev. Mol. Cell Biol. 16, 742-752 34. Lee, H.S. et al. (2015) Effects of N-glycosylation on protein conformation and dynamics: Protein Data Bank analysis and

molecular dynamics simulation study. Sci. Rep. 5, 8926

35. Zhu, W. et al. (2001) Cytoplasmic O-glycosylation prevents cell surface transport of E-cadherin during apoptosis. EMBO J. 20,

$5999-6007$

36. Geng, F. et al. (2012) Multiple post-translational modifications

regulate E-cadherin transport during apoptosis. J. Cell Sci. 125, 2615-2625

37. Hall, M.K. et al. (2013) Glycan structures contain information for the spatial arrangement of glycoproteins in the plasma mem- brane. PLoS ONE 8, e75013

38. Hall, M.K. et al. (2014) Cell surface N-glycans influence the level of functional E-cadherin at the cell-cell border. FEBS Open Bio. 4, 892-897

39. Hall, M.K. et al. (2016) Predominant expression of hybrid N- glycans has distinct cellular roles relative to complex and oligo- mannose N-glycans. Int. J. Mol. Sci. 17, 925

40. Shashikanth, N. et al. (2016) Kinetic leasurements reveal enhanced protein-protein interactions at intercellular junctions. Sci. Rep. 6, 23623

41. Langer, M.D. et al. (2012) N-Glycosylation alters cadherin- mediated intercellular binding kinetics. J. Cell Sci. 125, 2478-2485

42. Guo, H-B. et al. (2009) Regulation of homotypic cell-cell adhe- sion by branched Nglycosylation of $\mathrm{N}$-cadherin extracellular EC2 and EC3 domains. J. Biol. Chem. 284, 34986-34997

43. Vester-Christensen, M.B. et al. (2013) Mining the O-mannose glycoproteome reveals cadherins as major O-mannosylated gly- coproteins. Proc. Natl. Acad. Sci. U.S.A. 110, 21018-21023

44. Baenziger, J.U. (2013) O-Mannosylation of cadherins. Proc. Natl. Acad. Sci. U.S.A. 110, 2085820859

45. Lommel, M. et al. (2013) Protein O-mannosylation is crucial for E- cadherin-mediated cell adhesion. Proc. Natl. Acad. Sci. U.S.A. 110, 21024-21029

46. Winterhalter, P.R. et al. (2013) O-Glycosylation of the non-canon- ical T-cadherin from rabbit skeletal muscle by single mannose residues. FEBS Lett. 587, 3715-3721 
47. Pinho, S.S. et al. (2011) Modulation of E-cadherin function and dysfunction by N-glycosylation. Cell. Mol. Life. Sci. 68, 1011-1020

48. Liwosz, A. et al. (2006) N-Glycosylation affects the molecular organization and stability of Ecadherin junctions. J. Biol. Chem. 281, 23138-23149

49. Jamal, B.T. et al. (2009) N-Glycosylation status of E-cadherin controls cytoskeletal dynamics through the organization of dis- tinct b-catenin- and g-catenin-containing AJs. Cell Health Cytoskelet. 2009, 67-80

50. Nita-Lazar, M. et al. (2010) Hypoglycosylated E-cadherin promotes the assembly of tight junctions through the recruit- ment of $\mathrm{PP}_{2} \mathrm{~A}$ to adherens junctions. Exp. Cell Res. 316, 1871-1884

51. Taniguchi, N. and Kizuka, Y. (2015) Glycans and cancer: role of N-Glycans in cancer biomarker, progression and metastasis, and therapeutics. In Advances in Cancer Research (Richard, R.D. and Lauren, E.B., eds), pp. 11-51, Academic Press

52. Zhao, Y-P. et al. (2014) Decreased core-fucosylation contributes to malignancy in gastric cancer. PLoS ONE 9, e94536

53. Liu, L. et al. (2013) The identification and characterization of novel N-glycan-based biomarkers in gastric cancer. PLoS ONE 8, e77821

54. Osumi, D. et al. (2009) Core fucosylation of E-cadherin enhances cell-cell adhesion in human colon carcinoma WiDr cells. Cancer Sci. 100, 888-895

55. Hu, P. et al. (2008) E-Cadherin core fucosylation regulates nuclear b-catenin accumulation in lung cancer cells. Glycoconj. J. 25, 843-850

56. Shao, K. et al. (2016) Posttranslational modification of E-cadherin by core fucosylation regulates Src activation and induces epithe- lial-mesenchymal transition-like process in lung cancer cells. Glycobiology 26, 142-154

57. Wen, C-L. et al. (2012) Development of an AlphaLISA assay to quantify serum core-fucosylated E-cadherin as a metastatic lung adenocarcinoma biomarker. J. Proteomics 75, 3963-3976

58. Kizuka, Y. and Taniguchi, N. (2016) Enzymes for N-glycan branching and their genetic and nongenetic regulation in cancer. Biomolecules 6, 25

59. Buckhaults, P. et al. (1997) Transcriptional regulation of $\mathrm{N}$-ace- tylglucosaminyltransferase $\mathrm{V}$ by the src oncogene. J. Biol. Chem. 272, 19575-19581

6o. Huang, B. et al. (2014) Expression of $\mathrm{N}$-acetylglucosaminyltrans- ferase $\mathrm{V}$ in gastric cancer correlates with metastasis and prog- nosis. Int. J. Oncol. 44, 849-857

61. Murata, K. et al. (2000) Expression of $\mathrm{N}$-acetylglucosaminyltrans- ferase $\mathrm{V}$ in colorectal cancer correlates with metastasis and poor prognosis. Am. Assoc. Cancer Res. 6, 1772-1777 
62. Li, D. et al. (2008) Knockdown of Mgat5 inhibits breast cancer cell growth with activation of $\mathrm{CD}_{4}+\mathrm{T}$ cells and macrophages. J. Immunol. 180, 3158-3165

66. Wei, T. et al. (2011) Down-regulation of GnT-V inhibits nasopha- ryngeal carcinoma cell CNE-2 malignancy in vitro and in vivo. Cancer Lett. 309, 151-161

67. Granovsky, M. et al. (2000) Suppression of tumor growth and

metastasis in Mgat5-deficient mice. Nat. Med. 6, 306-312

68. Elola, M.T. et al. (2015) Assembly, organization and regulation of cell-surface receptors by lectin-glycan complexes. Biochem. J. 469, 1-16

69. Nabi, I.R. et al. (2015) The galectin lattice at a glance. J. Cell Sci. 128, 2213-2219

70. Pinho, S.S. et al. (2009) The role of $\mathrm{N}$-acetylglucosaminyltrans- ferase III and $\mathrm{V}$ in the posttranscriptional modifications of E- cadherin. Hum. Mol. Genet. 18, 2599-2608

71. Pinho, S.S. et al. (2013) E-Cadherin and adherens-junctions stability in gastric carcinoma: Functional implications of glycosyl- transferases involving $\mathrm{N}$-glycan branching biosynthesis, $\mathrm{N}$-acetylglucosaminyltransferases III and V. Biochim. Biophys. Acta (BBA) - Gen. Sub. 1830, 2690-2700

72. Carvalho, S. et al. (2016) Preventing E-cadherin aberrant N- glycosylation at Asn-554 improves its critical function in gastric cancer. Oncogene $35,1619-1631$

73. Pinho, S.S. and Reis, C.A. (2021) E-cadherin glycosylation in cancer. In Glycoscience: Biology and Medicine (Endo, T. et al. eds), pp. 1-6, Springer Japan

74. Guo, H-B. et al. (2003) N-Acetylglucosaminyltransferase V expression levels regulate cadherinassociated homotypic cell- cell adhesion and intracellular signaling pathways. J. Biol. Chem. 278, $52412-52424$

75. Zhao, H. et al. (2008) N-Glycosylation at Asn residues 554 and 566 of E-cadherin affects cell cycle progression through extra- cellular signal-regulated protein kinase signaling pathway. Acta Biochim. Biophys. Sin. (Shanghai). 40, 140-148

76. Zhao, H. et al. (2008) N-Glycosylation affects the adhesive func- tion of E-Cadherin through modifying the composition of adhe- rens junctions (AJs) in human breast carcinoma cell line MDAMB-435. J. Cell. Biochem. 104, 162-175

77. Varelas, X. et al. (2014) Protein N-glycosylation in oral cancer: dysregulated cellular networks among DPAGT1, E-cadherin adhe- sion and canonical Wnt signaling. Glycobiology 24, 579-591

78. Nita-Lazar, M. et al. (2009) Overexpression of DPAGT1 leads to aberrant N-glycosylation of Ecadherin and cellular discohesion in oral cancer. Cancer Res. 69, 5673-5680 
79. Zhou, F. et al. (2008) Unglycosylation at Asn-633 made extra- cellular domain of E-cadherin folded incorrectly and arrested in endoplasmic reticulum, then sequentially degraded by ERAD. Glycoconj. J. 25, 727-740

8o. Büll, C. et al. (2014) Sialic acids sweeten a tumor's life. Cancer Res. 74, 3199-3204

81. Dall'Olio, F. et al. (2014) Sialosignaling: sialyltransferases as engines of self-fueling loops in cancer progression. Biochim. Biophys. Acta Gen. Sub. 1840, 2752-2764

82. Varki, A. (2009) Essentials of Glycobiology, Cold Spring Harbor Laboratory Press

83. Langereis, M.A. et al. (2015) Complexity and diversity of the mammalian sialome revealed by nidovirus virolectins. Cell Rep. 11, 1966-1978

84. Pinho, S.S. et al. (2009) Molecular plasticity of E-cadherin and sialyl Lewis X expression, in two comparative models of mam- mary tumorigenesis. PLoS ONE 4, e6636

85. Lin, S. et al. (2002) Cell surface /2,6-sialylation affects adhesion of breast carcinoma cells. Exp. Cell Res. 276, 101-110

86. Schultz, M.J. et al. (2016) The tumor-associated glycosyltrans- ferase ST6Gal-I regulates stem cell transcription factors and confers a cancer stem cell phenotype. Cancer Res. 76, 3978-

63. Demetriou, M. et al. (1995) Reduced contact-inhibition and sub-

stratum adhesion in epithelial cells expressing GlcNAc-transfer-

ase V. J. Cell Biol. 130, 383-392 3988

64. Guo, H-B. et al. (2010) Specific posttranslational modification regulates early events in mammary carcinoma formation. Proc. Natl. Acad. Sci. U.S.A. 107, 21116-21121

65. Guo, H. et al. (2014) Post-translational glycoprotein modifications regulate colon cancer stem cells and colon adenoma progres- sion in Apcmin/+ mice through altered Wnt receptor signaling. J. Biol. Chem. 289, 31534-31549

87. Carvalho, S. et al. (2016) O-Mannosylation and N-glycosylation: two coordinated mechanisms regulating the tumour suppressor functions of E-cadherin in cancer. Oncotarget. Published online August 12, 2016. http://dx.doi.org/10.18632/oncotarget.11245

88. Jin, F-Z. et al. (2013) A correlation between altered O-GlcNAcy- lation, migration and with changes in E-cadherin levels in ovarian cancer cells. Exp. Cell Res. 319, 1482-1490

89. Chaiyawat, P. et al. (2014) Aberrant O-GlcNAcylated proteins: new perspectives in breast and colorectal cancer. Front. Endo- crinol. (Lausanne). 5, 193 
90. Saldova, R. et al. (2011) Core fucosylation and /2-3 sialylation in serum N-glycome is significantly increased in prostate cancer comparing to benign prostate hyperplasia. Glycobiology 21, 195-205

91. Zhao, Y. et al. (2014) Modification of sialylation mediates the invasive properties and chemosensitivity of human hepatocellular carcinoma. Mol. Cell. Proteomics 13, 520-536

92. Park, J-J. and Lee, M. (2013) Increasing the /2,6 sialylation of glycoproteins may contribute to metastatic spread and thera- peutic resistance in colorectal cancer. Gut. Liver 7, 629-641

93. Schultz, M.J. et al. (2012) Regulation of the metastatic cell phenotype by sialylated glycans. Cancer Metastasis Rev. 31, 501-518

94. Alpaugh, M.L. et al. (2002) Cooperative role of E-cadherin and sialyl-Lewis X/A-deficient MUC1 in the passive dissemination of tumor emboli in inflammatory breast carcinoma. Oncogene 21, $3631-3643$

95. Gomes, C. et al. (2013) Expression of $\mathrm{ST}_{3} \mathrm{GAL}_{4}$ leads to SLe(x) expression and induces c-Met Activation and an invasive phe- notype in gastric carcinoma cells. PLoS ONE 8, e66737

96. Bassagañas, S. et al. (2014) Pancreatic cancer cell glycosylation regulates cell adhesion and invasion through the modulation of /2b1 integrin and E-cadherin function. PLoS ONE 9, eg8595

97. Siddiqui, S.F. et al. (2005) Coexpression of b1,6-N-acetylgluco- saminyltransferase V glycoprotein substrates defines aggressive breast cancers with poor outcome. Cancer Epidemiol. Biomark- ers Prev. 14, 2517-2523

98. Huang, B. et al. (2013) Downregulation of the GnT-V gene inhibits metastasis and invasion of BGC823 gastric cancer cells. Oncol. Rep. 29, 2392-2400

99. Lee, J.H. et al. (2013) N-Acetylglucosaminyltransferase V triggers overexpression of MT1-MMP and reinforces the invasive/meta- static potential of cancer cells. Biochem. Biophys. Res. Commun. 431, 658-663

100. Kim, Y-S. et al. (2008) Functional proteomics study reveals that $\mathrm{N}$ acetylglucosaminyltransferase $\mathrm{V}$ reinforces the invasive/meta- static potential of colon cancer through aberrant glycosylation on tissue inhibitor of metalloproteinase. Mol. Cell. Proteomics 7, 114

101. Terao, M. et al. (2011) Enhanced epithelial-mesenchymal transi- tion-like phenotype in $\mathrm{N}$ acetylglucosaminyltransferase $\mathrm{V}$ trans- genic mouse skin promotes wound healing. J. Biol. Chem. $286,28303-28311$

102. Ihara, Y. et al. (1993) CDNA cloning, expression, and chromo- somal localization of human Nacetylglucosaminyltransferase III (GnT-III). J. Biochem. 113, 692-698

103. Nagae, M. et al. (2016) Atomic visualization of a flipped-back conformation of bisected glycans bound to specific lectins. Sci. Rep. 6, 22973 
104. Re, S. et al. (2011) Structural diversity and changes in confor- mational equilibria of biantennary complex-type $\mathrm{N}$-glycans in water revealed by replica-exchange molecular dynamics simula- tion. Biophys. J. 101, L44-L46

105. Taniguchi, N. et al. (1999) Implication of N-acetylglucosaminyltrans- ferases III and V in cancer: gene regulation and signaling mecha- nism. Biochim. Biophys. Acta Mol. Basis Dis. 1455, 287-300

106. Zhao, Y. et al. (2006) N-Acetylglucosaminyltransferase III antag- onizes the effect of Nacetylglucosaminyltransferase $\mathrm{V}$ on $/ 3 \mathrm{~b} 1$ integrin-mediated cell migration. J. Biol. Chem. 281, $32122-32130$

107. Song, Y. et al. (2010) The bisecting GlcNAc on N-glycans inhibits growth factor signaling and retards mammary tumor progres- sion. Cancer Res. 70, 3361-3371

108. Yoshimura, M. et al. (1995) Suppression of lung metastasis of B16 mouse melanoma by Nacetylglucosaminyltransferase III gene transfection. Proc. Natl. Acad. Sci. U.S.A. 92, 8754- 8758

109. Kitada, T. et al. (2001) The addition of bisecting $\mathrm{N}$-acetylglucos- amine residues to e-cadherin down-regulates the tyrosine phos- phorylation of b-catenin. J. Biol. Chem. 276, 475-480

110. Yoshimura, M. et al. (1996) Aberrant glycosylation of E-cadherin enhances cell-cell binding to suppress metastasis. J. Biol. Chem. 271, 13811-13815

111. Akama, R. et al. (2008) N-Acetylglucosaminyltransferase III expression is regulated by cell-cell adhesion via the E-cad- herin-catenin-actin complex. Proteomics 8, 3221-3228

112. lijima, J. et al. (2006) Cell-cell interaction-dependent regulation of $\mathrm{N}$ acetylglucosaminyltransferase III and the bisected N-glycans in GE11 epithelial cells: involvement of e-cadherin-mediated cell adhesion. J. Biol. Chem. 281, 13038-13046

113. $\mathrm{Xu}, \mathrm{Q}$. et al. (2011) Wnt/b-catenin signaling down-regulates $\mathrm{N}$ - acetylglucosaminyltransferase III Expression: the implications of two mutually exclusive pathways for regulation. J. Biol. Chem. $286,4310-4318$

114. Xu, Q. et al. (2012) Roles of N-acetylglucosaminyltransferase III in epithelial-to-Mesenchymal transition induced by transforming growth factor b1 (TGF-b1) in epithelial cell lines. J. Biol. Chem. $287,16563-16574$

115. Pinho, S.S. et al. (2012) Loss and recovery of Mgat3 and GnT-III mediated E-cadherin Nglycosylation is a mechanism involved in epithelial-mesenchymal-Epithelial Transitions. PLoS ONE 7, e33191

116. de Gramont, A. et al. (2015) Pragmatic issues in biomarker evaluation for targeted therapies in cancer. Nat. Rev. Clin. Oncol. 12, 197-212

117. Oliveira, C. et al. Familial gastric cancer: genetic susceptibility, pathology, and implications for management. Lancet Oncol. 16, e6o-70. 
118. Christgen, M. et al. (2016) Lobular breast cancer: clinical, molec- ular and morphological characteristics. Pathol. Res. Pract. 212, 583-597

119. Weiß, J.V. et al. (2011) Soluble E-cadherin as a serum biomarker candidate: Elevated levels in patients with late-stage colorectal carcinoma and FAP. Int. J. Cancer 128, 1384-1392

120. Mestre, T. et al. (2016) Quantification of topological features in cell meshes to explore Ecadherin dysfunction. Sci. Rep. 6, 25101

121. Kuefer, R. et al. (2003) The role of an $80 \mathrm{kDa}$ fragment of E- cadherin in the metastatic progression of prostate cancer. Am. Assoc. Cancer Res. 9, 6447-6452

122. Chan, A. et al. (2001) Soluble E-cadherin is a valid prognostic marker in gastric carcinoma. Gut $48,808-811$

123. Almeida, A. and Kolarich, D. (2016) The promise of protein glycosylation for personalised medicine. Biochim. Biophys. Acta Gen. Sub. 1860, 1583-1595

124. Mehta, N. et al. (2016) Mass spectrometric quantification of $\mathrm{N}$ - linked glycans by reference to exogenous standards. J. Prote- ome Res. Published online July 19, 2016. http://dx.doi.org/ 10.1021/acs.jproteome.6boo132

125. Fuster, M.M. and Esko, J.D. (2005) The sweet and sour of cancer: glycans as novel therapeutic targets. Nat. Rev. Cancer 5, 526-542

126. Kolarich, D. et al. (2012) Determination of site-specific glycan heterogeneity on glycoproteins. Nat. Protocols 7, 1285-1298

127. Patwa, T. et al. (2010) Glycoprotein analysis using protein micro- arrays and mass spectrometry. Mass Spectrom. Rev. 29, 830-844

128. Mereiter, S. et al. (2016) Glycomic and sialoproteomic data of gastric carcinoma cells overexpressing ST3GAL4. Data Brief 7, 814-833

129. Campos, D. et al. (2015) Probing the O-glycoproteome of gastric cancer cell lines for biomarker discovery. Mol. Cell. Proteomics 14, 1616-1629

130. Muthana, S.M. and Gildersleeve, J.C. (2016) Factors affecting anti-glycan IgG and IgM repertoires in human serum. Sci. Rep. 6, 19509

131. Pochechueva, T. et al. (2011) Comparison of printed glycan array, suspension array and ELISA in the detection of human anti-glycan antibodies. Glycoconj. J. 28, 507-517

132. Peng, W. et al. (2013) Glycan microarray screening assay for glycosyltransferase specificities. In Glycosyltransferases: Meth- ods and Protocols (Brockhausen, I., ed.), pp. 1-14, Humana Press 
133. Rillahan, C.D. and Paulson, J.C. (2011) Glycan microarrays for decoding the glycome. Annu. Rev. Biochem. 80, 797-823

134. Narimatsu, H. et al. (2010) A strategy for discovery of cancer glyco-biomarkers in serum using newly developed technologies for glycoproteomics. FEBS J. 277, 95-105

135. Ghirardello, M. et al. (2016) Glycomimetics targeting glycosyl- transferases: synthetic, computational and structural studies of less-polar conjugates. Chemistry 22, 7215-7224

141. Cummings, R.D. and Pierce, J.M. (2014) The challenge and promise of glycomics. Chem. Biol. 21, 1-15

142. Stanley, P. (2011) Golgi glycosylation. Cold Spring Harb. Per- spect. Biol. 3, aoo5199

143. Jensen, P.H. et al. (2010) Mucin-type O-glycosylation - putting the pieces together. FEBS J.

$277,81-94$

144. Neubert, P. and Strahl, S. (2016) Protein O-mannosylation in the early secretory pathway. Curr. Opin. Cell Biol. 41, 100-

136. Wu, Y. et al. (2010) Cooperativity between trans and cis inter- 108

actions in cadherin-mediated junction formation. Proc. Natl.

Acad. Sci. U.S.A. $107,17592-17597$

137. Zhang, Y. et al. (2009) Resolving cadherin interactions and bind-ing cooperativity at the singlemolecule level. Proc. Natl. Acad. Sci. U.S.A. 106, 109-114

138. Ishiyama, N. and Ikura, M. (2012) The three-dimensional struc- ture of the cadherin-catenin complex. In Adherens Junctions: from Molecular Mechanisms to Tissue Development and Disease (Harris, T., ed.), pp. 39-62, Netherlands, Springer

139. Nanes, B.A. and Kowalczyk, A.P. (2012) Adherens junction turnover: regulating adhesion through cadherin endocytosis, degradation, and recycling. Subcel. Biochem. 60, 197-222

140. Baum, B. and Georgiou, M. (2011) Dynamics of adherens junc- tions in epithelial establishment, maintenance, and remodeling. J. Cell Biol. 192, 907-917

145. Praissman, J.L. and Wells, L. (2014) Mammalian O-mannosyla- tion pathway: glycan structures, enzymes, and protein sub- strates. Biochemistry 53, 3066-3078

146. Ohtsubo, K. and Marth, J.D. (2006) Glycosylation in cellular mechanisms of health and disease. Cell 126, 855-867

147. Zhao, Y-Y. et al. (2008) Functional roles of N-glycans in cell signaling and cell adhesion in cancer. Cancer Sci. 99, 1304-1310 
148. Rabinovich, G.A. and Toscano, M.A. (2009) Turning 'sweet' on immunity: galectin-glycan interactions in immune tolerance and inflammation. Nat. Rev. Immunol. 9, 338-352

149. Blidner, A.G. et al. (2015) Re-wiring regulatory cell networks in immunity by galectin-glycan interactions. FEBS Lett. 589, 3407-3418 

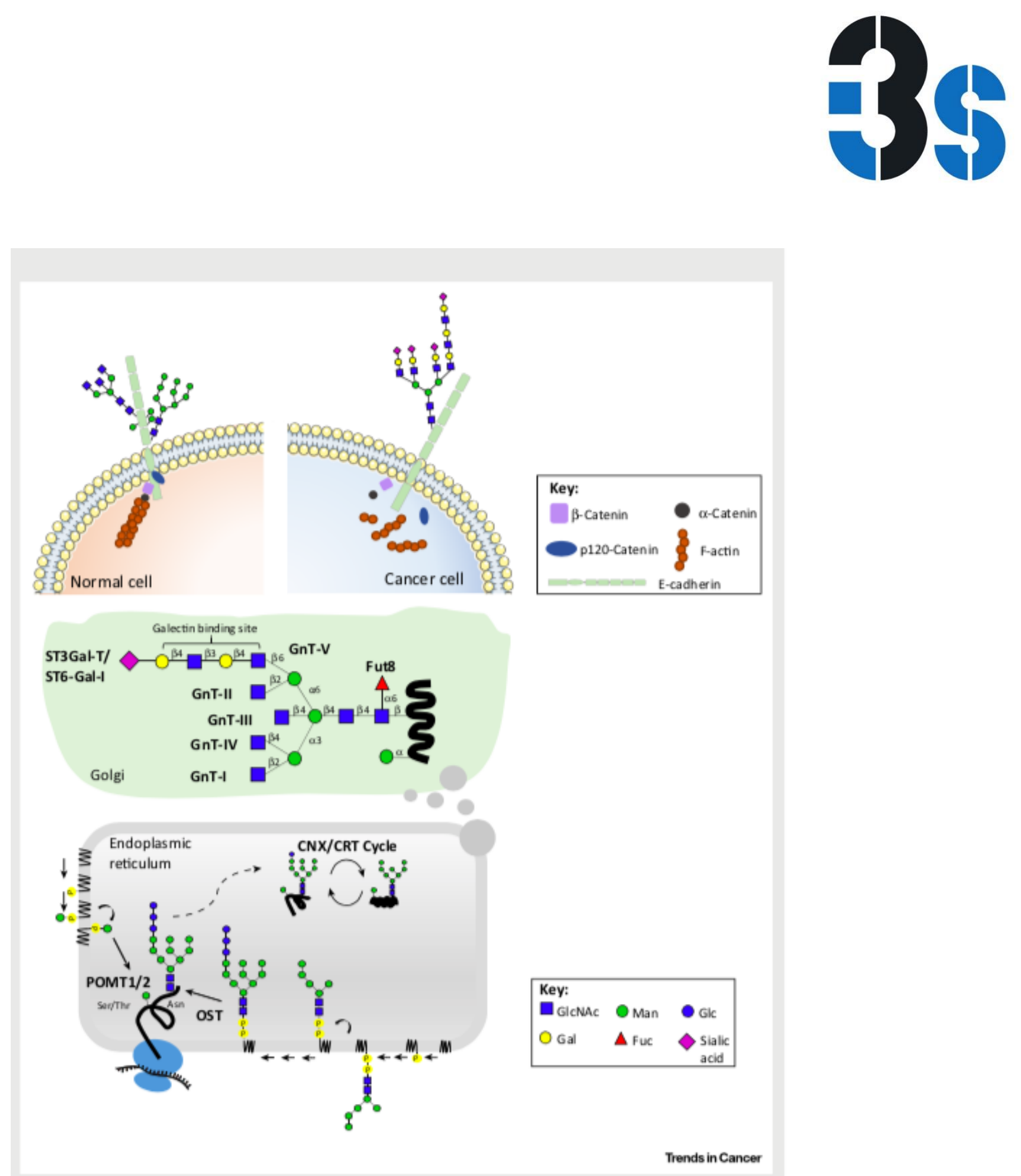

Figure I. The Glycosylation Pathway in Normal and Cancer Cells. Abbreviations: CNX, cainexin; CRT, calreticuln.

Rua Alfredo Allen, 208 4200-135 Porto Portugal $+351220408800$ 

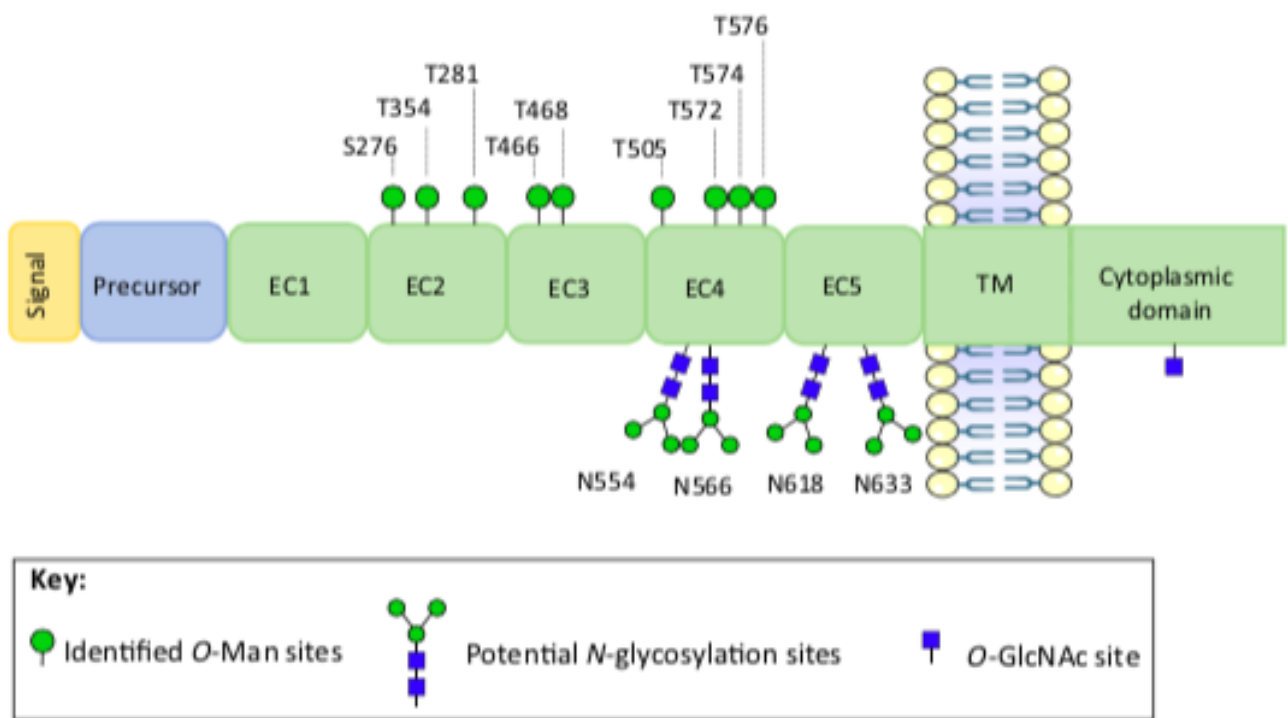

Trends in Cancer

Figure 1. Schematic Representation of Classical Cadherin Structure and Glycosylation Sites. The major forms of cadherin glycosylation that impact upon its cell-adhesion functions in cancer are $\mathrm{N}$ glycans, O-mannosyl glycans, and O- GlcNAc structures. The human E-cadherin ectodomain comprises four potential $\mathrm{N}$-glycosylation sites: two putative sites located in the $\mathrm{EC}_{4}$ subdomain (Asn-554 and Asn-566), and two sites in the EC5 subdomain (Asn-618 and Asn-633). The Nglycosylation of E-cadherin contributes to up to $20 \%$ of its total mass, and glycosylation site occupancy is dependent on cell, tissue, and pathophysiological context. The O-mannose glycoproteome recently identified classical cadherins as major carriers of O-linked mannose structures attached to potential and predicted glycosites localized in the EC2-5 domains. Moreover, O-GlcNAc glycans were also described to modify E-cadherin in its cytoplasmic domain, thereby interfering with its folding, trafficking, and consequently cell-adhesion functions. Abbreviations: EC, extracellular domain; O-GlcNAc; O- linked N-acetylglucosamine; O-Man, O-linked mannosylation; $\mathrm{TM}$, transmembrane domain. 


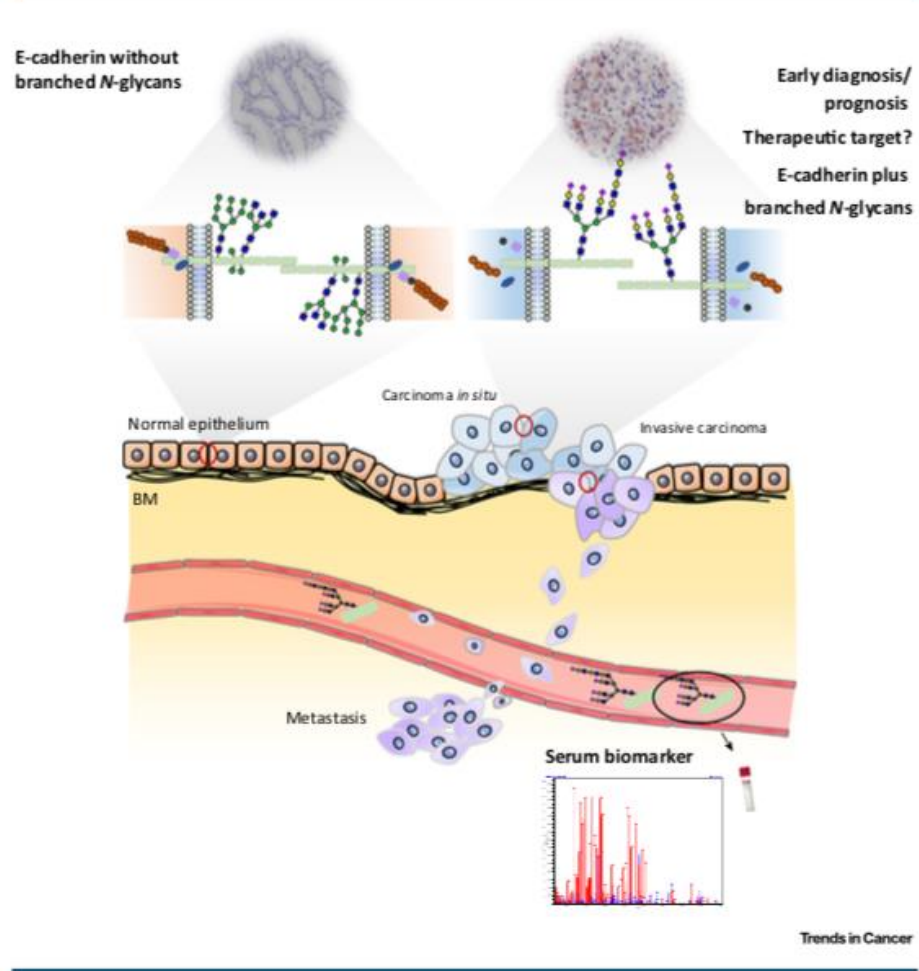

Figure 2. For a Figure36o author presentation of Figure 2, see the figure online at http://dx.doi.org/10.1016/j.trecan.2016. 08.003\#mmc1. Modifications in the glycosylation of cadherins occur during cellular transformation, invasion, and metastases. In home- ostasis and in normal/suppressive contexts, where cell-cell adhesion is preserved and adherens junctions are competent, E-cadherin is predominantly modified with bisecting $\mathrm{N}$-glycans, high-mannose $\mathrm{N}$ glycans, and O-mannosyl glycans. During neoplastic transformation, E-cadherin is highly modified with b1,6-GlcNAc branched N-glycans, further extended with poly- LAcNAc structures and terminal sialylation, that promote malignant and invasive phenotypes. These different glycoforms of cadherins are hallmarks of either 'normal' or cancer phenotypes, and can be used as biomarkers. The detection of cadherins specifically modified with b1,6-GlcNAc branched N-glycans in a biopsy sample or, as an non-invasive approach, in serum (by proximity ligation assay technology or glycan array) supports its applicability in cancer early diagnosis, determination of prognosis, and patient risk stratification. Abbreviation: BM, basement membrane. 